\title{
Photon correlation vs. interference of single-atom fluorescence in a half-cavity
}

\author{
François Dubin ${ }^{1}$, Daniel Rotter ${ }^{1}$, Manas Mukherjee $^{1}$, Carlos Russo $^{1}$, Jürgen Eschner ${ }^{2}$, and Rainer Blatt ${ }^{1 \dagger}$ \\ ${ }^{1}$ Institute for Experimental Physics, University of Innsbruck, Technikerstr. 25, A-6020 Innsbruck, Austria \\ ${ }^{2}$ ICFO - Institut de Ciències Fotòniques, 08860 Castelldefels (Barcelona), Spain. \\ ${ }^{\dagger}$ Institute for Quantum Optics and Quantum Information of the Austrian Academy of Sciences, 6020 Innsbruck, Austria
}

(Dated: August 22, 2018)

\begin{abstract}
Photon correlations are investigated for a single laser-excited ion trapped in front of a mirror. Varying the relative distance between the ion and the mirror, photon correlation statistics can be tuned smoothly from an antibunching minimum to a bunching-like maximum. Our analysis concerns the non-Markovian regime of the ion-mirror interaction and reveals the field establishment in a half-cavity interferometer.
\end{abstract}

PACS numbers: 32.80.-t, 42.50.Lc, 42.50.Ct, 42.50.Vk

Experiments with laser-cooled trapped ions have provided important contributions to the understanding of quantum phenomena. A single trapped ion is in fact a model system whose internal and external degrees of freedom can be controlled at the quantum level: non-classical motional states such as Fock states and quadraturesqueezed states have been successfully engineered with a single $\mathrm{Be}^{+}$ion [1]; the internal levels of trapped ions have been coherently manipulated by sequences of laser pulses, and have been entangled with the motional state, leading to the preparation of Schrödinger cat states [2] and to multi-ion entangled states for quantum information processing [3].

The internal dynamics of a laser-driven single ion or atom is well characterized by the statistical analysis of the measured stream of fluorescence photons, namely by the second order correlation function $G^{(2)}(T)$ [4], i.e. the frequency of time intervals of length $T$ between detected photons. For a single atom trapped in free space, this correlation function exhibits sub-Poissonian statistics and violates the Cauchy-Schwarz inequality, i.e. $G^{(2)}(0)<G^{(2)}(T)$. More precisely, $G^{(2)}(T)$ exhibits a minimum at $T=0$ which indicates the quantum nature of photon emission, or the projective character of photon detection. This is defined as anti-bunching [5, 6]. On the contrary, for a large ensemble of atoms the emitted radiation exhibits classical bunching [7] fulfilling $G^{(2)}(0) \geq$ $G^{(2)}(T)$. A smooth transition from anti-bunching to bunching has recently been observed in a high-Q resonator when increasing the number of interacting atoms [8].

The second order correlation function can be viewed as representing the (average) dynamics of the observed system conditioned on the emission of a photon at time $T=0$. While $G^{(2)}$ thereby draws on the photon character of the emitted light, it is the wave character which is responsible for interference phenomena, in particular for QED effects in resonators. In this letter, we examine the interplay of photon detection and wave interference in a simple cavity QED experiment, by measuring the second order photon correlation for a single trapped $\mathrm{Ba}^{+}$ion in a half-cavity interferometer. In this set-up part of the resonance fluorescence of the laser-excited ion is retro- reflected by a mirror at a distance $L$ and focussed back onto its source. Earlier experiments with our system revealed back-action of the interferometer on the emitting atom such as modification of its decay rate 9 and energy shifts of the excited state 10]; even mechanical action was observed [1]. Such effects intrinsically pertain to the interference caused by the mirror. On the other hand, the mirror induces a time delay $\tau=2 L / c$, needed for photons to return to the ion's position. When $\tau$ is negligible on the time scale of the atomic dynamics, the modified decay rate and energy shift correspond to the "low-Q" regime of cavity QED [12]. Here we investigate a different regime, when $\tau$ is comparable to the spontaneous emission lifetime. This characterizes a non-Markovian situation, where retardation and memory effects play a major role: the emitted photon projects the atom, and interference can only be established after the delay time $\tau$, when the atomic dynamics have already evolved significantly [13]. This problem was first discussed theoretically by Cook and Milonni 14], then by Alber [15], and recently by Dorner and Zoller [16] with a particular emphasis on our experimental conditions. Our study is, to our knowledge, the first single-atom implementation of such a system.

We report measurements for two ion-mirror distances, $L=67 \mathrm{~cm}$ and $90 \mathrm{~cm}$, and find them in quantitative agreement with theoretical predictions. Depending on the exact position of the mirror, which we vary on the nanometer scale, the $G^{(2)}$ function shows radically different behaviour. In particular, we observe how the interference in the mode reflected by the mirror sets in with the retardation time $\tau$. At a more general level, this corresponds to a sudden transition in the dynamics of the atom-cavity system from a regime where which-way information is present to the regime where interference is established. Moreover, through varying $L$, the value of $G^{(2)}(0)$ for our single atom can be tuned from an antibunching minimum to a bunching-like maximum.

The schematic experimental set-up and the relevant partial level scheme of ${ }^{138} \mathrm{Ba}^{+}$are shown in Fig.1. The ion is continuously driven and cooled by two narrow-band tunable lasers at $493 \mathrm{~nm}$ (green) and $650 \mathrm{~nm}$ (red) exciting the $\mathrm{S}_{1 / 2}-\mathrm{P}_{1 / 2}$ and $\mathrm{P}_{1 / 2}-\mathrm{D}_{3 / 2}$ transitions, respec- 


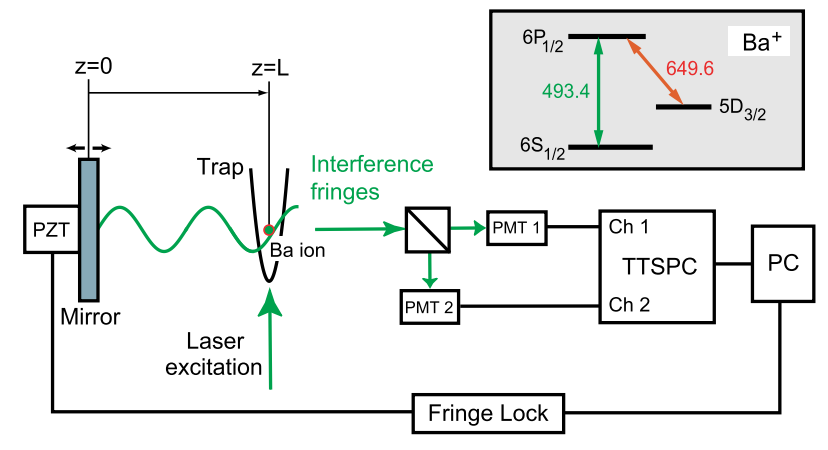

FIG. 1: A single ${ }^{138} \mathrm{Ba}^{+}$ion in a Paul trap (parabola) is continuously laser-excited. A lens (not shown) and a mirror at distance $L$, mounted on piezo-actuators (PZT), focus back part of the fluorescence onto the ion. Green (493 nm) photons are detected by two photomultipliers (PMT 1 and 2) and their arrival times are correlated with 100 ps temporal resolution (TTSPC: Time Tagged Single Photon Counting). A slow electronic servo loop (fringe lock) stabilises the average photocurrent and thereby permits control of the distance $L$ between the ion and the mirror with better than $10 \mathrm{~nm}$ precision.

tively. Laser frequencies are close to resonance and intensities are set below saturation. A fraction $\epsilon$ of the green fluorescence photons is reflected by a distant mirror and focussed back onto the ion. We analyse the $G^{(2)}$ correlation function of the $493 \mathrm{~nm}$ light in the observation channel opposite to the mirror. This light has two components, the direct and the reflected part of the radiation scattered by the ion, with a time delay $\tau$ between them. For very low laser intensities, when all scattering is elastic, the resulting interference of these components is observed with up to $72 \%$ visibility [9] into that mode. In the measurements presented here we use slightly higher laser excitation rate, whereby the contrast reduces to around $50 \%$. The interference signal can be viewed as a consequence of the standing wave which forms in the mirror mode and which leads to inhibited and enhanced detection of resonance fluorescence photons [9]. The signal varies with the ion-mirror distance $L$ as $\sin ^{2}\left(k_{f l} L\right)$, where $k_{f l}$ is the momentum of photons emitted at $493 \mathrm{~nm}$. A fringe minimum corresponds to the ion being located at a node of the standing wave, i.e. $k_{f l} L=n \pi$ ( $n$ being an integer); the maximum corresponds to $k_{f l} L=\left(n+\frac{1}{2}\right) \pi$, i.e. to the ion being at an antinode.

We note that on average there are less than $10^{-3}$ photons in the mode volume between the ion and the mirror. This gives rise to one of the remarkable features of this experiment, that the interference is created by partial waves corresponding to the same individual photon, while at the same time the detection of these photons reveals dynamical information and state projection of the atom.

We now study the second order correlation for arrival times of green photons. First we recall the main theoret- ical results of Ref.[16], restricting the treatment to the $\mathrm{S}_{1 / 2}$ and $\mathrm{P}_{1 / 2}$ levels. As shown in Fig $\square$ we label the mirror-ion-detector axis as $z$, set the mirror position at $z=0$ and the trap center at $z=L$. Neglecting the motion of the ion in the trap, the field operator for green photons in the mirror mode reads at $z=L$

$$
\begin{gathered}
E_{m}(L, t)=\frac{\epsilon \Gamma}{2} \frac{i \hbar}{d} e^{-i \omega_{L} t}\left[\sigma^{-}(t) \theta(t)\right. \\
\left.-e^{i \omega_{L} \tau} \sigma^{-}(t-\tau) \theta(t-\tau)\right]+N_{v}(t),
\end{gathered}
$$

where $\theta(t)$ is a step function centered at $t=0, \Gamma$ is the free-space decay rate of the $\mathrm{P}_{1 / 2}$ to $\mathrm{S}_{1 / 2}$ transition, and $d$ its dipole oscillator strength. $\sigma^{-}$denotes the lowering operator from $\left|\mathrm{P}_{1 / 2}\right\rangle$ to $\left|\mathrm{S}_{1 / 2}\right\rangle$ and $\omega_{L}$ the laser frequency. $N_{v}$ is the source free part of the mirror field, i.e. the input state in the language of input-output theory [17]. In Eq. (1) the interaction picture with respect to the free part of the Hamiltonian is used, operators become time dependent, and we turn into a frame rotating at the laser frequency, e.g. $\sigma^{-}(t) \rightarrow \sigma^{-}(t) e^{-i \omega_{L} t}$. Including proper commutation rules between input and output states of the field, the second order time correlation function in the mirror mode, $G_{m}^{(2)}(t, t+T)=\left\langle E_{m}^{\dagger}(L, t) E_{m}^{\dagger}(L, t+\right.$ $\left.T) E_{m}(L, t+T) E_{m}(L, t)\right\rangle$, reads

$$
\begin{array}{r}
G_{m}^{(2)}(t, t+T) \propto \| \sigma^{-}(t+T) \sigma^{-}(t) \\
+e^{2 i \omega_{L} \tau} \sigma^{-}(t+T-\tau) \sigma^{-}(t-\tau) \\
-\mathcal{T}_{\hookleftarrow} e^{i \omega_{L} \tau} \sigma^{-}(t+T-\tau) \sigma^{-}(t) \\
-e^{i \omega_{L} \tau} \sigma^{-}(t+T) \sigma^{-}(t-\tau)|i\rangle \|^{2},
\end{array}
$$

where $|i\rangle$ denotes the initial state of the system, i.e. the ion in the ground state $\left|S_{1 / 2}\right\rangle$ and the mirror mode in the vacuum state. The different contributions in Eq.(2) are interpreted as follows: the first term corresponds to the detection of two photons directly emitted towards the detectors and separated by a time interval $T$; in the second term, these photons are both reflected by the mirror (therefore delayed by $\tau$ ) before detection. The two last contributions describe possible detection of either first a directly emitted photon and then a second one after its reflection on the mirror (third term), or vice-versa (fourth term). In the former case, for $T<\tau$ causality is ensured by $\mathcal{T}_{\hookleftarrow}$ which enforces the time ordering of the two operators on its right hand side. These must be arranged chronologically from right to left and have to be commuted if they are not. Consequently, in Eq.(2) different contributions interfere. The first two terms induce anti-bunching around $T=0$ while the two others may counteract this usual behavior. As we show below, the weight of each component strongly depends on the actual position of the ion, i.e. wether it is located at a node or at an anti-node of the mirror mode. Finally, from Eq.(2) one obtains in the steady-state limit $(t \rightarrow \infty)$

$$
\begin{aligned}
& G_{m}^{(2)}(T) \propto \mid 2 b_{\mathrm{P}_{1 / 2}}(T) \cos \left(2 k_{f l} L\right) \\
& -b_{\mathrm{P}_{1 / 2}}(|T-\tau|)-\left.b_{\mathrm{P}_{1 / 2}}(T+\tau)\right|^{2}
\end{aligned}
$$



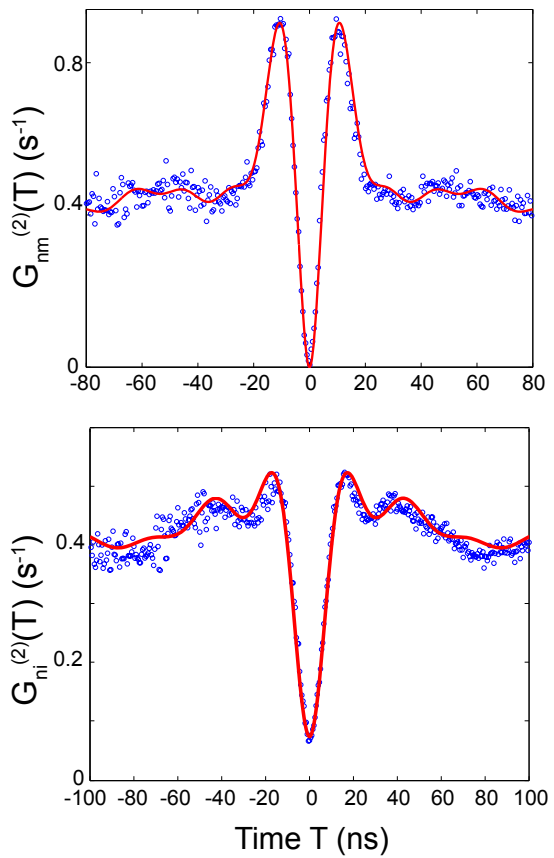

FIG. 2: Top: Measured second order correlation function without mirror, $G_{n m}^{(2)}$ (circles) and its simulation calculated from 8-level Bloch equations (line). Bottom: Correlation function for non-interfering ion and mirror image, $G_{n i}^{(2)}$, for $\tau=4.5 \mathrm{~ns}$. The line is the sum of three correlation functions as explained in the text. For the measured curves we evaluate the time intervals between all pairs of detected photons using a 500 ps time bin width, and then divide the data by the total integration time (several hours) after background subtraction.

where $b_{\mathrm{P}_{1 / 2}}$ denotes the occupation amplitude of the $\mathrm{P}_{1 / 2}$ level. In principle, it should be evaluated including the mirror induced modifications of decay rate and energy value of the $\mathrm{P}_{1 / 2}$ state [9, 10, 11]. Nevertheless, the mirror back-action can be neglected for the current analysis, with $\epsilon$ being on the order of $1.5 \%$. Then $b_{\mathrm{P}_{1 / 2}}$ is deduced from the density matrix time evolution considering a single $\mathrm{Ba}^{+}$ion trapped in free space. Note that all 8 electronic sub-levels need to be accounted for in order to accurately reproduce the exact shape of the measured correlations [18].

In the top panel of Fig 2 we present the correlation function in absence of the mirror, $G_{n m}^{(2)}$. It is obtained using the set-up depicted in Fig प but with the mirror blocked. The measurement exhibits the characteristic anti-bunching at short time, with a null rate of coincidences, $G_{n m}^{(2)}(0) \simeq 0$. It is accurately reproduced by our simulations which do not require any fitting parameter, only experimental conditions such as laser powers and detunings [18]. The lower panel of Fig 20 shows the correlation function when the mirror is included, but without overlapping the reflected field with its source; ion and mirror image are then spatially distinct, and there is no interference. The signal, $G_{n i}^{(2)}(T)$, corresponds to three synchronous but non-interfering sources, shifted in time

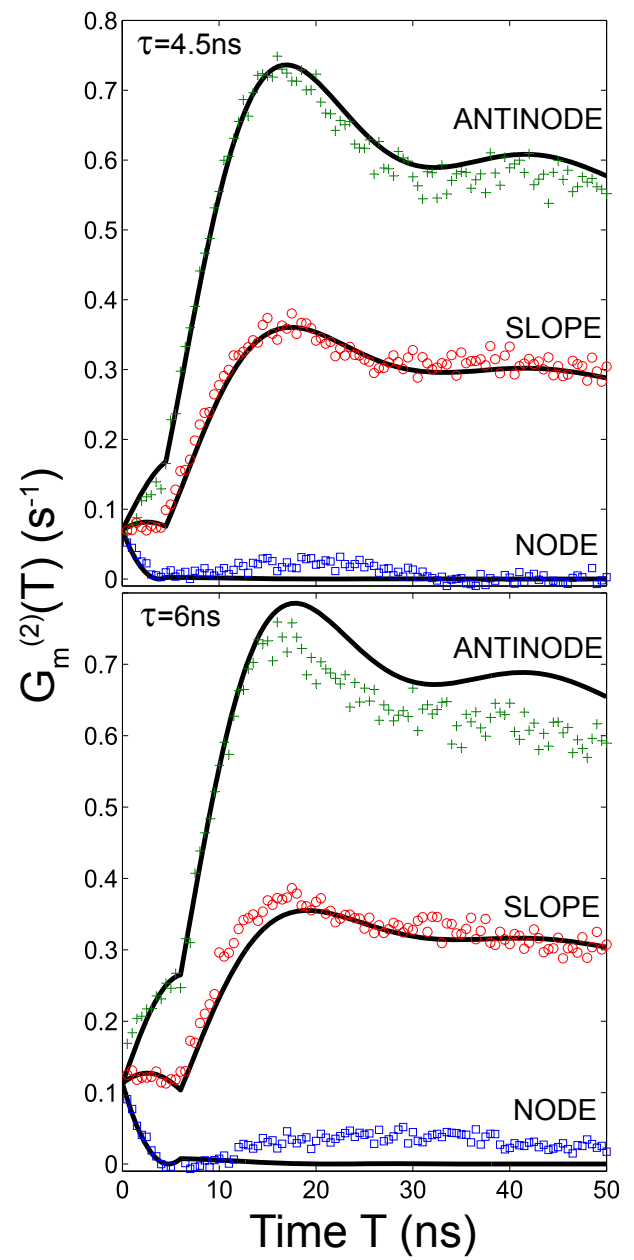

FIG. 3: Measured correlation function $G_{m}^{(2)}(T)$, after subtraction of the non-interfering part, for the ion placed near a node (squares), a slope (circles) and an anti-node (crosses) of the standing-wave mirror mode. Each data set corresponds to 3 hours of integration. The lines represent the results of our model (Eq.(3)).

by $\pm \tau$. The expected contributions to this signal are the moduli squares of the three terms in Eq.(3), without the cosine dependance, i.e. without interference. As shown by the full line, their sum accurately reproduces our measurements. In the following this signal is used as a reference: in the model leading to Eq.(3), experimental conditions are assumed ideal with $100 \%$ fringe contrast of the green interference. Experimentally a contrast of 50\% is observed, such that Eq. (3) only accounts for half of the measured correlations, while the remaining part corresponds to $G_{n i}^{(2)}$. Therefore in all data sets for $G_{m}^{(2)}(T)$ shown below, the measured $G_{n i}^{(2)}(T)$ has already been subtracted from the raw histogram data.

Figure 3 presents such measured second order correlation functions $G_{m}^{(2)}(T)$ for interfering ion and mirror image. We compare three relevant situations: the ion close 
to a node $\left(k_{f l} L=0.03 \pi\right)$, on the slope $\left(k_{f l} L=0.28 \pi\right)$ and close to an antinode $\left(k_{f l} L=0.4 \pi\right)$ of the standingwave mirror mode. The first notable feature is that always $G_{m}^{(2)}(0)>0$. For our single trapped ion, such coincidence can only appear when a directly emitted and reflected photon are simultaneously detected. This is possible in our experiment since the delay of a reflected photon is comparable to the time required to re-excite the ion to the $\mathrm{P}_{1 / 2}$ state. The second important feature is that all situations show the same coincidence rate $G_{m}^{(2)}(0)$, although the relative phase $\left(2 k_{f l} L\right)$ between the coincident direct and reflected photon fields is different in the three situations. This demonstrates that at $T=0$ one has the full which-way information about the two photons. Consequently no interference can be observed.

We now discuss the long-time limit $T \gg \tau$ : in Eq. (3) the time argument of $b_{\mathrm{P}_{1 / 2}}$ reduces to $\mathrm{T}$ and

$G_{m}^{(2)}(T) \equiv \sin ^{4}\left(k_{f l} L\right)\left|b_{\mathrm{P}_{1 / 2}}(T)\right|^{2}\left|b_{\mathrm{P}_{1 / 2}}^{(s s)}\right|^{2},\left|b_{\mathrm{P}_{1 / 2}}^{(s s)}\right|^{2}$ being the steady state population of the $\mathrm{P}_{1 / 2}$ state. The second order correlation function thus factorizes into the product of the first order correlations at time $t$ and $(t+T)$. For the anti-node position, the interference is constructive and $G_{m}^{(2)}(T \gg \tau)$ is maximal. On the other hand, at the node position the fully established destructive interference suppresses the detection of photon pairs with long time intervals between them, thus creating a strong effective bunching around $\mathrm{T}=0$ despite the fact that we are dealing with only a single atom.

Finally we study the correlations for short time delay between photon detections, $0<T \leq \tau$. In this regime memory effects are crucial, as one can see from Eq.(3), where excited-state amplitudes at different times are superimposed. The difference between the three positions originates mainly from the weight $\cos \left(2 k_{f l} L\right)$ of the first term in Eq.(3), which corresponds to the processes where both photons are emitted in the same direction. The two other terms, describing processes where they take opposite directions, do not depend on the mirror phase. As a result, a conspicuous kink in all the curves at $T \approx \tau$ is observed. This kink marks the sudden onset of full interference, when no more which-way information is present.

To summarize, for a single ion trapped and laserexcited in front of a mirror, we have presented the second order time correlation function of emitted photons. Depending on the position of the ion, e.g. at a node or at an antinode of the reflected field standing wave, very different behaviours are shown for large distances between the ion and the mirror. In this non-Markovian regime, the detection of photon pairs separated by a large time interval is modulated by the interference experienced by each photon. On the other hand, coincident two photon detections are insensitive to the exact position of the ion, because interference can not be established and which way information for each detected photon is accessible. Consequently, when the ion is placed at a node of its reflected fluorescence standing wave, a single photon detection is prohibited by first order interference while a joint two photon detection is allowed. This appears as a bunched profile in the correlation function which reveals the transient regime of the field establishment in our half cavity interferometer. We believe that our analysis characterizes the transient regime of cavity quantum electrodynamics.

This work has been partially supported by the Austrian Science Fund (project SFB15), by the European Commission (QUEST network, HPRNCT-2000-00121, QUBITS network, IST-1999-13021, SCALA Integrated Project, Contract No. 015714), by a travel grant of the ÖAD (No. 3/2005), the Spanish MEC (No. HU2004-0015) and by the "Institut für Quanteninformation GmbH."
[1] D. M. Meekhof et al., Phys. Rev. Lett 76, 1796 (1996)

[2] C. Monroe et al., Science 272, 1131 (1996)

[3] H. Häffner et al., Nature 438, 643 (2005)

[4] L. Mandel, E. Wolf, Optical Coherence and Quantum Optics (Cambridge University Press, U.S., 1995).

[5] J.H. Kimble et al., Phys. Rev. Lett 39, 691 (1977)

[6] F. Diedrich et al., Phys. Rev. Lett 58, 203 (1987); M. Schubert et al., Phys. Rev. Lett 68, 3016 (1992)

[7] R. Hanbury Brown and R. Q. Twiss, Nature (London) 1781046 (1956)

[8] M. Hennrich et al., Phys. Rev. Lett 94, 053604 (2005)

[9] J. Eschner et al., Nature 413, 495 (2001).

[10] M. A. Wilson et al., Phys. Rev. Lett. 91, 213602 (2003).
[11] P. Bushev et al., Phys. Rev. Lett. 92, 223602 (2004).

[12] P. Milonni, The quantum vacuum (Academic Press, London, 1993).

[13] Photon correlations in the Markovian regime will be reported elsewhere: D. Rotter et al., in preparation.

[14] R.J. Cook et al., Phys. Rev. A 35, 5081 (1987).

[15] G. Alber, Phys. Rev. A 46, R5338-R5341 (1992).

[16] U. Dorner et al., Phys. Rev. A 66, 23816 (2002).

[17] C. W. Gardiner and P. Zoller, Quantum Noise (Springer, Berlin, 2004).

[18] M. Schubert et al., Phys. Rev. A 52, 2994 (1995). 